\title{
Leveraging Sensor Fusion and Sensor-Body Position for Activity Recognition for Wearable Mobile Technologies
}

\author{
https://doi.org/10.3991/ijim.v15i17.25197 \\ Ashraful Alam, Anik Das, Md. Shahriar Tasjid, Ahmed Al Marouf $f^{(凶)}$ \\ Daffodil International University, Dhaka, Bangladesh \\ marouf.csediu.edu.bd
}

\begin{abstract}
Smart devices like smartphones and smartwatches have made this world smarter. These wearable devices are created through complex research methodologies to make them more usable and interactive with its user. Various interactive mobile applications such as augmented reality (AR), virtual reality (VR) or mixed reality (MR) applications solely depend on the in-built sensors of the smart devices. A lot of facilities can be taken from these devices with sensors such as accelerometer and gyroscope. Different physical activities such as walking, jogging, sitting, etc., can be important for analysis like health state prediction and duration of exercise by using those sensors based on artificial intelligence. In this paper, we have implemented machine learning and deep learning algorithms to detect and recognize eight activities namely, walking, jogging, standing, walking upstairs, walking downstairs, sitting, sitting-in-a-car and cycling; with a maximum of $99.3 \%$ accuracy. A few activities are almost similar in action, such as sitting and sitting-in-a-car, but difficult to distinguish; which makes it more challenging to predict tasks. In this paper, we have hypothesized that with more sensors (sensor fusion) and data collection points (sensor-body positions) a wide range of activities can be recognized and the recognition accuracies can be increased. Finally, we showed that the combination of all the sensors data of both pocket/waist and wrist can be used to recognize a wide range of activities accurately. The possibility of using the proposed methodologies for futuristic mobile technologies is quite significant. The adaptation of most recent deep learning algorithms such as convolutional neural network (CNN) and bi-directional Long Short Time Memory (Bi-LSTM) demonstrated high credibility of the methods presented as experimentation.
\end{abstract}

Keywords—-sensor fusion, human activity recognition, machine learning, deep learning, sensors

\section{Introduction}

We are living in the age of technological advancements. In this era, the field of human-centric computing research is an emerging field of research in which we can 
understand the nature of human behavior, habit, interests, etc. Human activity recognition is a type of work where all we have to do is to recognize or predict human movement by studying and analyzing human-computer interaction (HCI), video surveillance, wearable devices, or sensors. However, the challenging part here is to detect, predict activities with decent accuracy. In our work, we tried to recognize human activity by using in-built sensors of smartphones. Sensors give high-frequency data every second with the physical movement of different parts of the body. Keeping smartphones in the pocket will not give us the same data if it is kept in hand. Thus, it is a challenging task to predict human activities from a wide range of sensor data precisely.

Human activity recognition can be defined as "determining or recognizing the human activity using technological aspects". In literature, we found there are several ways to recognize human activities, such as sitting, walking, running, walking upstairs, walking downstairs, etc. Among the technological aspects, use of image processing [1-4] has been widely accepted and the reviews [5-9] have shown the various techniques focusing on the prediction task. Feature extraction and dimensionality reduction-based approaches were used to get significant performance by the classifiers. The sensor-based approaches $[2,6]$ are not new in this area, but the usability of the smartphone in-built sensors is still in research.

For this paper, we have placed one smartphone on the wrist and another one in the pocket to capture data of different activities. The sensors utilized for this task are accelerometer and gyroscope for each of the smartphones. The contributions of the papers are listed:

- Integration of multiple sensor data into one multi-model dataset by sensor fusion technique and utilize it for the further processing.

- Consideration of sensor-body positions such as wrist, pocket and wrist-pocket to collect sensor data and utilize it for the further processing.

- Applying classification algorithms e.g., KNN, CNN, Bi-directional LSTM, SVM, etc. for recognizing the different activities.

We have collected data for sitting in car and cycling, which are novel in the domain and integrating these activities will provide robustness in the training-testing method.

\section{$2 \quad$ Related works}

There has been intense research going on for the past 2 decades on the area of human computer interaction, particularly on human activity recognition. Several attempts have been made to detect Human Activity from different body points attached to the bodies. Here [10] accelerometer and microphone data were used to recognize activities in a certain environment. Their attempt was to get good accuracy even if the device's location in the body changes. Another attempt here [11] used only a tri-axial accelerometer of a phone to detect activities. They performed their experiment keeping the phone both in wrist and pocket. Then both model's accuracy was compared. They used both individual and combinations of classifiers. Their results were promising. Vinh [12] used the 
data of the accelerometer from both hip and waist. Their attempt was to detect activity using low-powered and low-cost devices. Bao [13] used bi-axial accelerometer data retrieved from wrist, ankle, thigh, elbow, and hip. While collecting data they did not ask the users, where they should put the device while doing the activities. Therefore, the data came up from those five different body locations. After testing with several algorithms and comparing them, they proved that only thigh and waist data combined can perform close to the five data points combined. The decision tree was the best classifier in their experiment. Jatoba [14] and his team's work has been done for monitoring activities on patients. So, they analyzed the data of micro accelerometer placed on the patient's chest. With KNN and CART, they were able to get decent accuracy.

Most of the work has been done on the accelerometer data taken from either waist or wrist. But Zhu [15] used accelerometer data from foot and waist. And their best-performed algorithm was HMM. They reduced the complexity of the dataset by fusion of the data collected from foot and waist. This way they overcame the problem of the need for a strong displacement of sensors for the HMM model to work well. Some of the attempts were made in a discriminative way too [16]. The closest work to us is the work of San-Segundo-Hernández [17] and E. Bulbul [18]. In [17], they used accelerometer data from the wrist and pocket. They claimed accelerometer data provided better results compared to that of the gyroscope. What we have done is that, instead of analyzing the data of the accelerometer and gyroscope separately, we treated both sensor data as features for output and trained our model from that data. This way a wide range of activities can be measured accurately as the model gets more features to work on. We proved this by analyzing different combination of sensors data in section 3.2. Bulbul [18] in their work, used both the sensors together to build the model. He achieved very good results too. But they used only pocket data. Our work includes both the sensors from two different devices placed on the pocket and wrist. This way the model can be trained to successfully distinguish among wide ranges of different activities even if they are quite similar like sitting in a moving car or a chair in a stationary place. Similarly, Hip-joint based hand activity recognition was proposed in [38], where the distance between the hip-joint and the hand joints were extracted using the Microsoft Kinect skeleton tracking system.

Different experiments used different sensors placed on single or multiple places of body locations. There are lots of algorithms that are implemented, but few of them perform well enough. For example, SVM, ANN, HMM, CNN, LSTM, etc. It is evident from the comparison above that when different sensors are combined together as features or more than one sensor are placed in multiple places of the human body, the performance and accuracy are achieved better [33]. In the context of artificial intelligence, utilizing big data for green and sustainable technologies such as human activity recognition models are very crucial and important [34-35]. For the betterment of health technologies, merging of big data and green technologies is a must and the significance can be found in [36-37].

Machine learning algorithms, especially classification algorithms are utilized for similar to human activity recognition. Some of the significant problems addressed using the machine learning algorithms are: detecting malicious links from world 
wide web (www) [21], handwritten digit recognition [22], depression detection from image and video analysis [23], air temperature prediction [24], etc. Therefore, we have applied seven different classification algorithms namely, logistic regression, random forest, K- nearest neighbor (k-NN), Support vector machine (SVM), Gradient Boosting, Convolutional Neural Network (CNN), Bi-directional LSTM. The experimental results coming from these classifiers are highlighted in the result and analysis section.

Table 1. Comparative analysis and summary of different approaches made to detect human activity

\begin{tabular}{|l|l|l|l|c|}
\hline \multicolumn{1}{|c|}{ Paper } & \multicolumn{1}{|c|}{ Sensors } & \multicolumn{1}{|c|}{ Sensor Placed } & \multicolumn{1}{|c|}{ Algorithms } & $\begin{array}{c}\text { Best Accuracy } \\
\text { Obtained }\end{array}$ \\
\hline Lester [10] & $\begin{array}{l}\text { Accelerometer, } \\
\text { Microphone }\end{array}$ & $\begin{array}{l}\text { Waist, wrist, } \\
\text { shoulder }\end{array}$ & HHM & $90 \%$ \\
\hline Akram Bayat [11] & Accelerometer & $\begin{array}{l}\text { Either wrist or } \\
\text { waist }\end{array}$ & $\begin{array}{l}\text { SVM, LMT, } \\
\text { Random Forest }\end{array}$ & $91.15 \%$ \\
\hline E. Bulbul [18] & $\begin{array}{l}\text { Accelerometer, } \\
\text { Gyroscope }\end{array}$ & Pocket & $\begin{array}{l}\text { SVM, k-NN, } \\
\text { Bagging, Stacking }\end{array}$ & $99.4 \%$ \\
\hline $\begin{array}{l}\text { San-Segundo- } \\
\text { Hernández [17] }\end{array}$ & Accelerometer & Pocket, wrist & $\begin{array}{l}\text { CNN-LSTM, } \\
\text { HMM }\end{array}$ & $99.4 \%$ \\
\hline L. Vinh [12] & Accelerometer & Waist, hip & SMCRF & $88.38 \%$ \\
\hline C. Zhu [15] & Accelerometer & Foot, waist & HMM & $90 \%$ \\
\hline L. Bao [13] & Accelerometer & $\begin{array}{l}\text { Wrist, ankle, } \\
\text { thigh, elbow, hip }\end{array}$ & KNN, NB & $84 \%$ \\
\hline L. C. Jatoba [10] & Accelerometer, SPI & Chest & KNN, CART & $95 \%$ \\
\hline Z. He [19] & Accelerometer & Pocket & SVM & $97.51 \%$ \\
\hline A. Khan [20] & Accelerometer & Chest & ANN & $97.9 \%$ \\
\hline
\end{tabular}

\section{Proposed methodology}

\subsection{Research subject and instrument}

Nowadays, every person has at least one smartphone in their pocket. Along with that, people have been starting to wear wristbands instead of analog watches. These gadgets have different kinds of sensors built-in, especially accelerometers and gyroscopes which are common. There is a lot of usage of these two sensors. In our paper, we are focusing on human activity detection and recognition by using accelerometer and gyroscope sensors data. Here we have detected eight activities and these are walking, standing, sitting, upstairs, downstairs, jogging, cycling, and sitting in the car. We have collected this dataset by using three android operating system-based smartphones. These were Samsung Galaxy S10 Plus, Redmi Note 7s, and Huawei Y9. We have used an application called "Sensor Data" to collect our required data. 


\subsection{Data collection procedure}

Collecting high-frequency data properly was a challenging task. At first, we managed 17 volunteers to perform different activities for a specific time period. The android application which we used to collect data was set up at a $50 \mathrm{~Hz}$ sampling rate. So, data was recorded in the dataset in a rate of 50 samples per second. We used two smartphones at a time. One was in the pocket and the other was tied to the wrist. The device tied to the wrist can get similar data to a wristwatch. With those activities, we got data from two locations (wrist and pocket) for each of the activities. Each of them has both accelerometer and gyroscope sensor data which is 3 dimensional with data points known as axes $(\mathrm{x}, \mathrm{y}, \mathrm{z})$. So, we got total of 12 columns for those 4 sensors each having 3 axes. Finally, we have stored each distinct type of data such as 'Activity Hand_accelerometer', 'Activity_Hand_gyroscope', 'Activity_Pocket_accelerometer', 'Activity_Pocket_gyroscope' into text files. That is the procedure of our time series data collection. After finishing the data collection phase, we have pre-processed the raw data to make a suitable dataset where we can implement different algorithms. First of all, we have removed unnecessary strings from the text files. Because the mobile application generated some irrelevant strings at the beginning of the text file. Then we have converted each text file into a comma-separated value (CSV) file. Here, we have 12 input columns for each of the activities and 1 output column where we have labelled the activity name. And for each of the activities, we have taken 44,000 samples. After that, we have merged all the CSV files into one to get our complete dataset. In our dataset, we have got a total 3,52,000 samples or instances. These instances are fed into the next step for pre-processing.

\subsection{Data pre-processing}

In feature engineering step, number of different steps were needed for data manipulation. we removed null values by using mean and median methods. There were noises in the dataset which need to be removed. So, we have done filtering by the butterfly method to remove the noises from the dataset and make the dataset smooth. Then we have done label encoding. As our output label was categorical data, we had to convert that into numerical data by using the label encoding function. Furthermore, we have split our dataset into input and output columns. Along with this, we also split our dataset into a train set and test set at the ratio of $4: 1$, which is $80 \%-20 \%$ distribution. The test set was kept aside for final testing stage. In training stage K-Fold cross validation was used to train the models. This method is evident and widely accepted in the machine learning research community.

After splitting the dataset, we have implemented feature scaling on our dataset to bring all the data points into a specific range. We used Robust Scalar and Standard Scalar to scale the dataset. We have implemented both scalar fitting and transformation on the training dataset but only implemented transformation on the test dataset. This prevents data leakage. So, the test set remains completely unseen to the model.

As we have time-series data, we had to specify a specific sized window which is also known as a sliding window. We have tested different window sizes like 2 seconds, 
4 seconds and observed the performance. Choosing the window size is tricky. The bigger the window, the better the result will be. But too big of a window will overfit the model. Moreover, the processing will be heavy as in each window there are lots of data. This will cause more problems if activities are detected in real-time. On the other hand, smaller window will be very fast for processing, but the result will not be as good as comparatively larger window. Considering this trade-off, we used a window size of 4 seconds. We have also defined the hop size which is known as stride size. Therefore, sliding window keeps moving forward according to the stride size and in each step, it creates a sample of an activity, dimension of $200 \times 12$ each. Used hop size was large enough to reduce overlapping. It ensures the diversity of samples in each window.

Using the sliding window in our dataset, we have got 3D data. As mentioned earlier, each second 50 rows of data are recorded. Therefore, for a window of 4 seconds, we got 200 rows and 12 columns of data. Each window represents an activity. This is a perfect data shape for models that uses Neural Networks. So, we used this dataset for our CNN and Bi-Directional LSTM models.

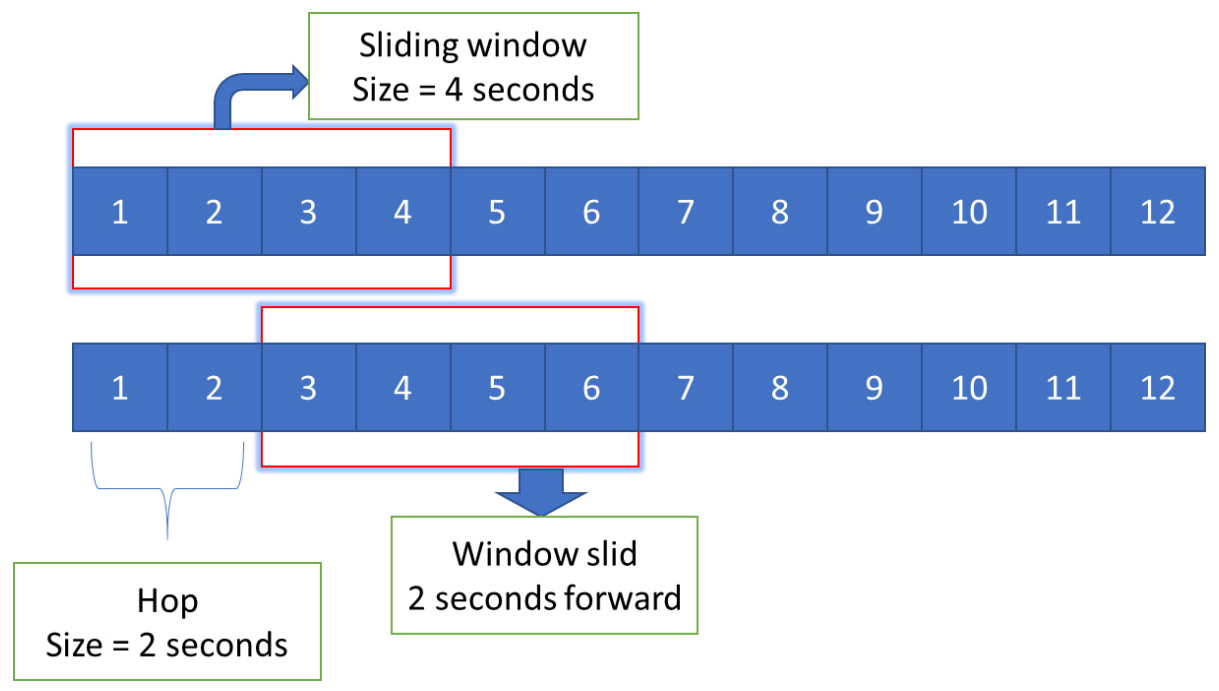

Fig. 1. Visualization of how sliding window was used for data overlapping for creation of the dataset

Therefore, for traditional machine learning models, we needed to flatten the dataset. Each activity's shape is $200 \times 12$ before flattening. Now after we flatten this, we get 2,400 columns for each activity. There are so many columns after flattening which can lead to overfitting. So, for reducing dimensionality, we used Principal Component Analysis (PCA).

And in the feature scaling step there were also some problems with $3 \mathrm{D}$ data while implementing the Deep Learning models. Therefore, we have also made an alternative option to reshape the whole training dataset. For doing that, we had to go through all the samples of activities and then reshape them. This is the complete procedure of our data collection, data storing, data cleaning, and data pre-processing. 

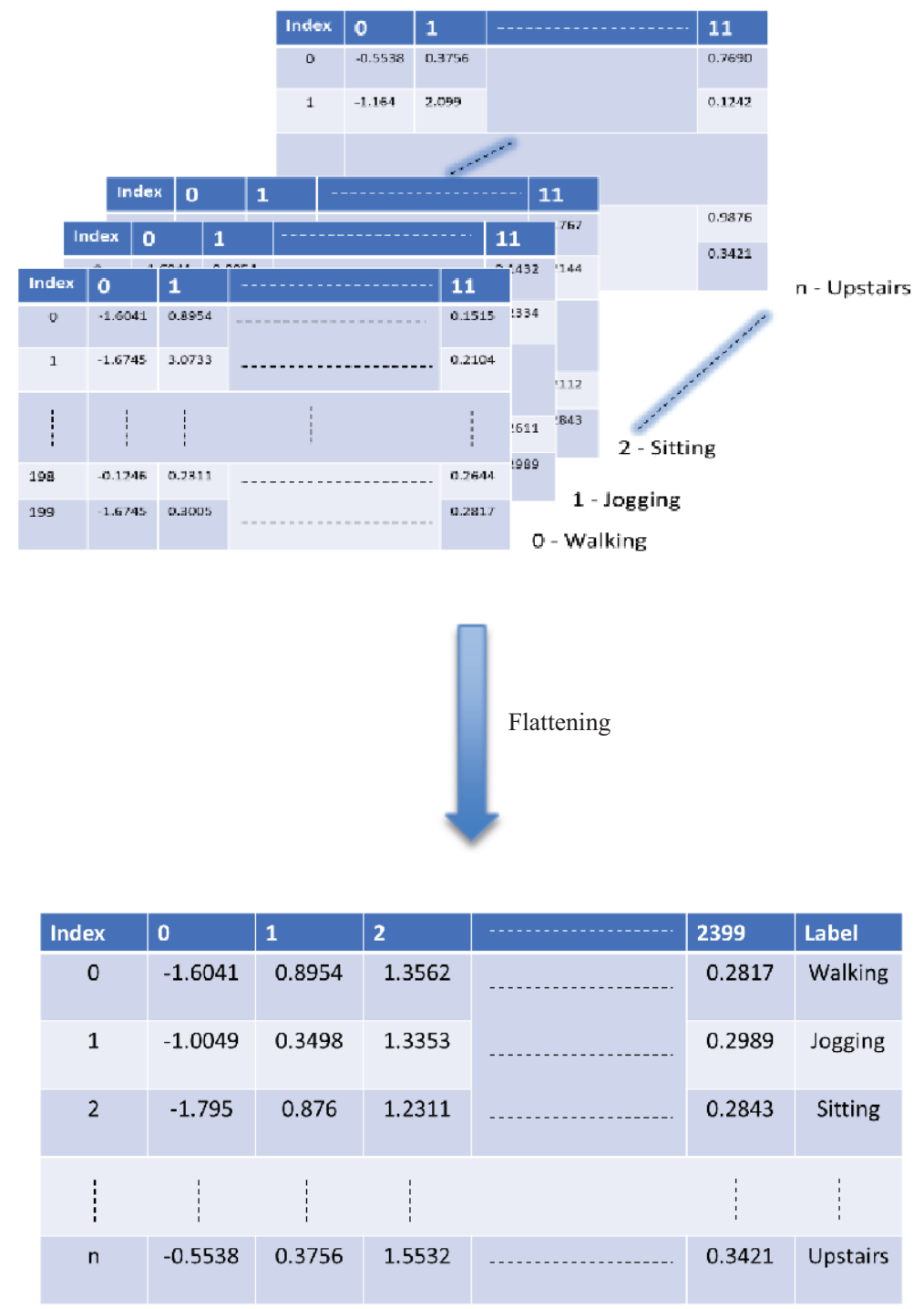

Fig. 2. How three-dimensional data is flattened into two-dimensional data

\section{$4 \quad$ Data analysis}

While performing any kind of activity, the orientation of the phone and smartwatch is expected to differ in different kinds of activities. And in most cases, this difference does occur. But often two or more activities can provide similar kinds of data. That means the data points will overlap in the same cartesian region. In that case, models can make an error to predict. This can be seen from the Figure 3 visualization of the 
data where some of the data are plotted. Looking at the accelerometer data of pocket, downstairs and upstairs data have similar kinds of dispersion, range, and orientation in some regions.
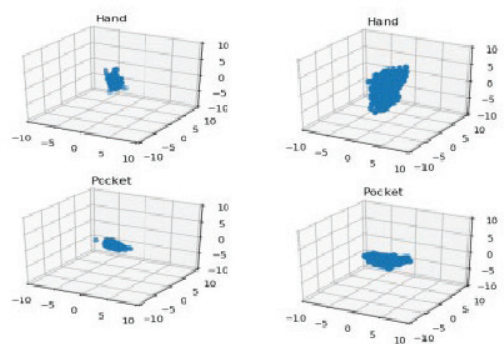

Walking Upstairs
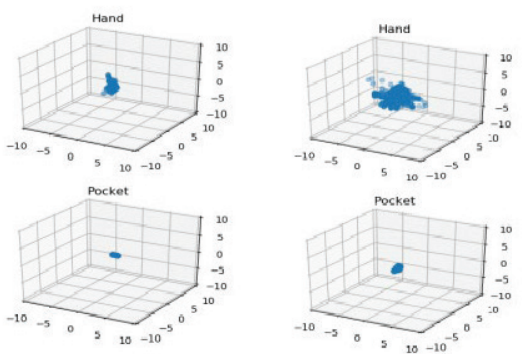

Sitting
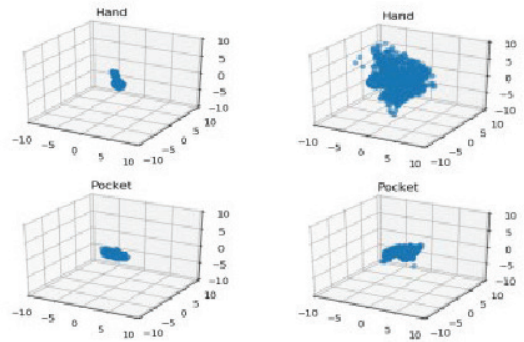

Walking Downstairs
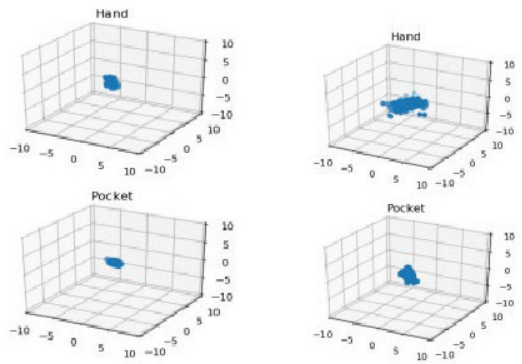

Sitting in a Car

Fig. 3. Comparative visualization of Accelerometer and Gyroscope data points in different activities

Therefore, Machine Learning algorithms might often struggle to distinguish between those activities. In cases like these, wrist data adds more variation. Though this is very hard to prove in visualization, so we can only have the intuition here. But in the 3.2 section, this hypothesis is proved by analyzing different sensor combination and it is proved that highest accuracy is reached when all the sensor data is taken as features. Looking at the wrist data of the accelerometer, data defers in dispersion and orientation in upstairs and downstairs activities. Adding the gyroscope to that, we will be able to add more information that can very easily be distinguished by the model. From the visualization of gyroscope data, we can see that both upstairs and downstairs data differ a lot. So visually, with these 4 sensors ( 2 in wrist and 2 in the waist) the margin of error seems to be very low for any activity that provides almost similar kind of data.

Sitting in a car and sitting home in a chair is an almost similar activity in action and hard to distinguish between those. From the Figure 3 visualization, we can see that accelerometer data of those 2 activities is hard to differentiate between. Data points have almost the same dispersion and orientation. Therefore, any machine learning algorithm will have plenty of errors when trying to predict if someone is sitting in a car or 
in a chair at home. In this scenario too, the gyroscope data adds variation that can easily be differentiated.
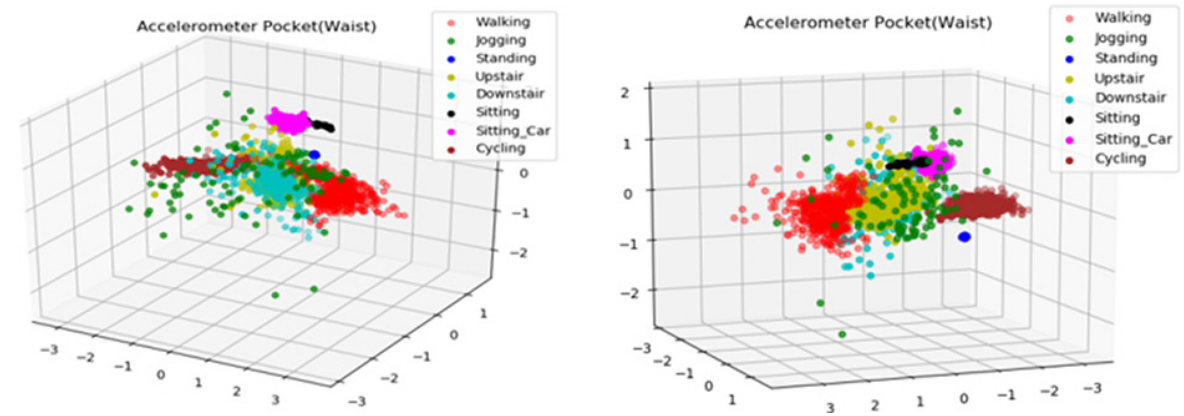

Fig. 4. Views from different angles of Accelerometer data recorded from the waist of 8 activities
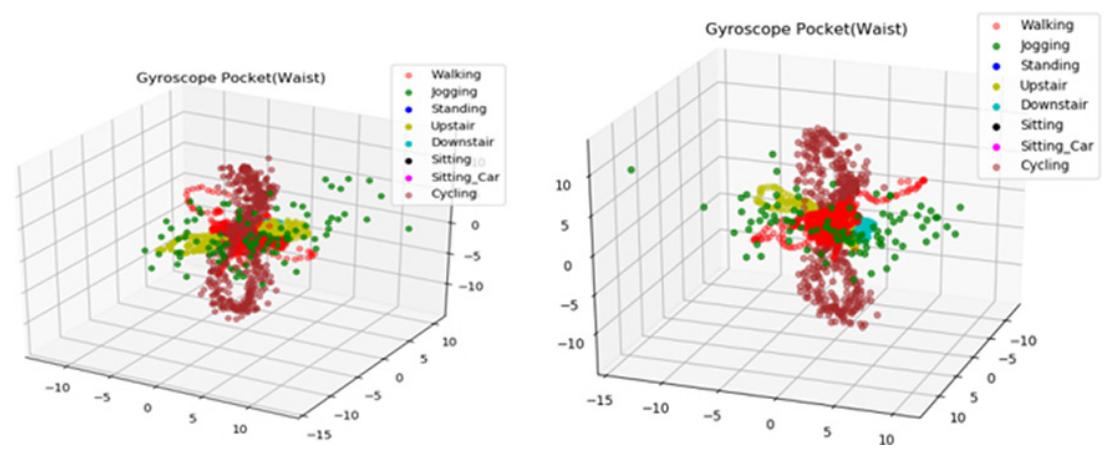

Fig. 5. Views from different angles of Gyroscope data recorded from the waist of 8 activities

From the above two scenarios, we can see when data are analyzed together from waist accelerometer, gyroscope, and wrist accelerometer, gyroscope we can predict the activities very accurately even if they are very similar in action. When all the activities are put together in a single graph, we can further visualize the above-mentioned problem. Here only waist data are plotted. It is enough to give an intuition for wrist data as well.

All the data plotted in these 2 figures (Figure 4, Figure 5), are the data of the same instance. In other words, these are data for specific 10 seconds from the entire dataset so that we can compare how the data points reside for different activities.

We can clearly see that upstairs and downstairs data are overlapping in Figure 4 and Figure 5 which are Yellow and Cyan respectively. So even from those 3 sensors, we might often not be able to distinguish between Upstairs and Downstairs. Some errors will come up eventually. But from Figure 5, we can easily distinguish between Cyan and Yellow. Therefore, these activities will be separated quite accurately. In the same way, other activities that are almost similar will be differentiated with those 4 activities even if they are almost similar in action. That is the reason why we are able to achieve an accuracy of $99.3 \%$ even though some of the activities are very similar to each other. 


\section{Experimental results and discussion}

In the past two decades, there have been many attempts to recognize human activities with precision. From our experiment, we will show that, when we combine the accelerometer and gyroscope data from pocket and wrist in columns as features, it is possible to detect and recognize a wide variety of activities with precision. The dataset we built and experimented with proves the base of our hypothesis. We have made the comparisons among accuracies obtained from different combinations of sensors and different combinations of positioning of devices if not used together. It will be evident how the accuracy gets affected if we remove one or more sensors or one device from our model. Here, by lower bound we mean the lowest accuracy by a specific model among the eight activities and by upper bound we mean the highest accuracy among the eight activities.

When the accelerometer and gyroscope data are used from a device placed in the pocket, we can see in Table 2, most of the algorithms perform well achieving 96\% to $97 \%$ accuracy. But if we look carefully, the Lower bound score did not reach up to the expected point. Though it is better than recognition of human activities from accelerometer and gyroscope sensor data from the device placed on the wrist, we still can improve the result by applying fusion on all the data. So, the models here do not perform very well in our dataset which we built very casually mimicking the reallife actions. Among the traditional classification algorithms, random forest has shown (Table 3) quite satisfactory performance, in terms of accuracy. It is evident that in many cases [25] similar to this, random forest is a good model. Though SVM has been proven to work better in some special cases, such as fault classification in smart distribution network [26], ozone prediction [27], cyberbullying identification [28], harmonic source identification [29], etc.

Table 2. Performance evaluation metrics using both sensors in Wrist and Pocket

\begin{tabular}{|l|c|c|c|c|c|c|}
\hline \multirow{2}{*}{ Algorithms } & \multicolumn{2}{|c|}{ Location: Wrist, Sensors: Both } & \multicolumn{2}{c|}{ Location: Pocket, Sensors: Both } \\
\cline { 2 - 7 } & $\begin{array}{c}\text { Lower } \\
\text { Bound } \\
\text { (f1-score) }\end{array}$ & $\begin{array}{c}\text { Upper } \\
\text { Bound } \\
\text { (f1-score) }\end{array}$ & Accuracy & $\begin{array}{c}\text { Lower } \\
\text { Bound } \\
\text { (f1-score) }\end{array}$ & $\begin{array}{c}\text { Upper } \\
\text { Bound } \\
\text { (f1-score) }\end{array}$ & Accuracy \\
\hline Logistic Regression & 0.08 & 0.96 & $63 \%$ & 0.47 & 1.00 & $83 \%$ \\
\hline Random Forest & 0.76 & 0.98 & $90 \%$ & 0.89 & 1.00 & $96 \%$ \\
\hline KNN & 0.63 & 1.00 & $85 \%$ & 0.89 & 1.00 & $95 \%$ \\
\hline SVM & 0.72 & 1.00 & $87 \%$ & 0.90 & 1.00 & $96 \%$ \\
\hline $\begin{array}{l}\text { Gradient Boosting } \\
\text { Classifier }\end{array}$ & 0.77 & 0.99 & $90 \%$ & 0.93 & 1.00 & $97 \%$ \\
\hline CNN & 0.66 & 0.99 & $84 \%$ & 0.88 & 1.00 & $96 \%$ \\
\hline Bi-LSTM & 0.08 & 0.96 & $63 \%$ & 0.47 & 1.00 & $83 \%$ \\
\hline
\end{tabular}

In Table 2, we can see that CNN did good. It has predicted all the activities with decent accuracy. But on the individual activity level, we can see that the lowest f1-score is only 0.77 when the device is placed at wrist. Therefore, it is evident that though some 
activities were detected quite accurately as the upper bound $\mathrm{f} 1$-score is up to 1.00 but to detect some activities $\mathrm{CNN}$ did struggle. Though $\mathrm{CNN}$ is an extremely powerful model and has shown quite satisfactory performance in many cases, such as detection of license plates [30], rice false smut detection [31], etc. So, the decision from Table 2 is, to predict different activities accurately, data from only wrist or pocket is not sufficient.

Table 3. Performance evaluation metrics using single sensor in both the locations

\begin{tabular}{|l|c|c|c|c|c|c|}
\hline \multirow{2}{*}{ Algorithms } & \multicolumn{2}{|c|}{$\begin{array}{c}\text { Location: Wrist \& Pocket; } \\
\text { Sensor: Accelerometer }\end{array}$} & \multicolumn{2}{c|}{$\begin{array}{c}\text { Location: Wrist \& Pocket; } \\
\text { Sensor: Gyroscope }\end{array}$} \\
\cline { 2 - 7 } & $\begin{array}{c}\text { Lower } \\
\text { Bound } \\
\text { (f1-score) }\end{array}$ & $\begin{array}{c}\text { Upper } \\
\text { Bound } \\
\text { (f1-score) }\end{array}$ & Accuracy & $\begin{array}{c}\text { Lower } \\
\text { Bound } \\
\text { (f1-score) }\end{array}$ & $\begin{array}{c}\text { Upper } \\
\text { Bound } \\
\text { (f1-score) }\end{array}$ & Accuracy \\
\hline Logistic Regression & 0.46 & 1.00 & $79 \%$ & 0.23 & 0.61 & $41 \%$ \\
\hline Random Forest & 0.84 & 1.00 & $95 \%$ & 0.74 & 0.99 & $86 \%$ \\
\hline KNN & 0.74 & 1.00 & $92 \%$ & 0.62 & 1.00 & $76 \%$ \\
\hline SVM & 0.64 & 1.00 & $86 \%$ & 0.03 & 0.99 & $72 \%$ \\
\hline $\begin{array}{l}\text { Gradient Boosting } \\
\text { Classifier }\end{array}$ & 0.78 & 1.00 & $93 \%$ & 0.71 & 1.00 & $86 \%$ \\
\hline CNN & 0.87 & 1.00 & $95 \%$ & 0.88 & 1.00 & $93 \%$ \\
\hline $\begin{array}{l}\text { Bi-directional } \\
\text { LSTM }\end{array}$ & 0.86 & 1.00 & $94 \%$ & 0.85 & 1.00 & $91 \%$ \\
\hline
\end{tabular}

If only accelerometer data is used (Table 3) from two devices placed in the pocket and wrist the models do not improve at all. Here best-performed algorithms are CNN and Random Forest. Though some activities were recognized without almost any error as the upper f1-score is perfect 1.00, their Lower bound f1-scores are still very low. But our aim is to recognize all the activities with the least margin of error.

Same goes for the models when gyroscope data is analyzed from those two locations. They are in no way any better than the previously analyzed situations. But what we can understand from these scenarios is, if we put all the data together, they will add up additional information when the data overlap for two or more activities in one sensor or device. Therefore, the accuracy will improve as we claim in our hypothesis.

Table 4. Performance evaluation (using all the devices and sensors together. Device location: Wrist and pocket; Sensor used: Accelerometer and Gyroscope)

\begin{tabular}{|l|c|c|c|}
\hline \multicolumn{1}{|c|}{ Algorithms } & $\begin{array}{c}\text { Lower Bound } \\
\text { (f1-score) }\end{array}$ & $\begin{array}{c}\text { Upper Bound } \\
\text { (f1-score) }\end{array}$ & Accuracy \\
\hline Logistic Regression & 0.60 & 0.99 & $84.2 \%$ \\
\hline Random Forest & 0.89 & 1.00 & $95.8 \%$ \\
\hline KNN & 0.74 & 1.00 & $90.7 \%$ \\
\hline SVM & 0.90 & 1.00 & $96.7 \%$ \\
\hline Gradient Boosting Classifier & 0.85 & 1.00 & $95.0 \%$ \\
\hline CNN & 0.94 & 1.00 & $98.2 \%$ \\
\hline Bi-LSTM & 0.99 & 1.00 & $99.3 \%$ \\
\hline
\end{tabular}


To prove our point, finally, the models are built using accelerometer and gyroscope data taken from pocket and wrist altogether as features. Which provides 12 features in total. Looking at Table 3, it can be easily seen that all the models scaled up a lot. Our best-performed model is Bi-directional LSTM. It has a lower bound f1-score of 0.99 which is very accurate and perfect for a model that can recognize human activities with very much precision. In our dataset, we took data of activities that are very similar in action and hard to distinguish between. For example, sitting in a chair at home, sitting in a car both are very similar in action. Sitting and standing are very stationary in action. But all those were recognized very accurately by Bi-directional LSTM.

\section{Conclusion}

Activities can be recognized in different ways using different algorithms and sensors placing the devices in different places of the human body. But the aim is how accurately we can recognize activity and most importantly if we can detect complex activities that might be similar in actions. We have introduced an optimal way to recognize the activity by using accelerometer and gyroscope sensors from the pocket and wrist. This helped us to accurately identify the eight activities where some of them are difficult to distinguish between for the similarities among them. Different traditional and Deep learning algorithms have been applied to our dataset as found useful in several machine learning related works [39-42]. Among them, Bi-directional LSTM gives optimal accuracy. Bidirectional LSTM gives us $99.3 \%$ of accuracy which is comparatively better. Thus, the idea of sensor fusion prevails and sensor-body position mechanism has given quite significant results, in terms of accuracy. The prospects of our proposed methods could be applied in health-related solutions. For example, diabetes patient's wearable sensors [32] could be fused and sensor-body positions may affect the proper recognition of health conditions.

\section{$7 \quad$ References}

[1] Soleimani, Elnaz, and Ehsan Nazerfard. "Cross-subject transfer learning in human activity recognition systems using generative adversarial networks.” Neurocomputing 426 (2021): 26-34. https://doi.org/10.1016/j.neucom.2020.10.056

[2] Erdaş, Çağatay Berke, and Selda Güney. "Human Activity Recognition by Using Different Deep Learning Approaches for Wearable Sensors." Neural Processing Letters (2021): 1-15. https://doi.org/10.1007/s11063-021-10448-3

[3] Tasnim, Nusrat, Mohammad Khairul Islam, and Joong-Hwan Baek. "Deep Learning Based Human Activity Recognition Using Spatio-Temporal Image Formation of Skeleton Joints." Applied Sciences 11, no. 6 (2021): 2675. https://doi.org/10.3390/app11062675

[4] Ke, Shian-Ru, Hoang Le Uyen Thuc, Yong-Jin Lee, Jenq-Neng Hwang, Jang-Hee Yoo, and Kyoung-Ho Choi. "A review on video-based human activity recognition." Computers 2 , no. 2 (2013): 88-131. https://doi.org/10.3390/computers2020088

[5] Ramanujam, E., Thinagaran Perumal, and S. Padmavathi. "Human Activity Recognition with Smartphone and Wearable Sensors using Deep Learning Techniques: A Review." IEEE Sensors Journal (2021). https://doi.org/10.1109/JSEN.2021.3069927

[6] Abdel-Salam, Reem, Rana Mostafa, and Mayada Hadhood. "Human Activity Recognition using Wearable Sensors: Review, Challenges, Evaluation Benchmark." arXiv preprint arXiv:2101.01665 (2021). https://doi.org/10.1007/978-981-16-0575-8_ 1 
[7] Straczkiewicz, Marcin, Peter James, and Jukka-Pekka Onnela. "A systematic review of smartphone-based human activity recognition for health research."

[8] Vrigkas, Michalis, Christophoros Nikou, and Ioannis A. Kakadiaris. "A review of human activity recognition methods." Frontiers in Robotics and AI 2 (2015): 28. https://doi. org/10.3389/frobt.2015.00028

[9] Aggarwal, Jake K., and Lu Xia. "Human activity recognition from 3d data: A review." Pattern Recognition Letters 48 (2014): 70-80. https://doi.org/10.1016/j.patrec.2014.04.011

[10] Lester, Jonathan and Choudhury, Tanzeem and Borriello, Gaetano, "A practical approach to recognizing physical activities, Pervasive Computing," Springer-Verlag Berlin Heidelberg, vol. 3968, pp. 1-16, 2006. https://doi.org/10.1007/11748625_1

[11] Akram Bayat, Marc Pomplun, Duc A. Tran, "A Study on Human Activity Recognition Using Accelerometer Data from Smartphones," Elsevier B.V, vol. 34, Pages 450-457, MobiSPC-2014. https://doi.org/10.1016/j.procs.2014.07.009

[12] L. Vinh, S. Lee, H. Le, H. Ngo, H. Kim, M. Han, and Y.-K. Lee, "Semi-markov conditional random fields for accelerometer-based activity recognition," Applied Intelligence, vol. 35, pp. 226-241, 2011. https://doi.org/10.1007/s10489-010-0216-5

[13] L. Bao and S. S. Intille, "Activity recognition from user-annotated acceleration data," in Pervasive, pp. 1-17, 2004. https://doi.org/10.1007/978-3-540-24646-6 1

[14] L. C. Jatoba, U. Grossmann, C. Kunze, J. Ottenbacher, and W. Stork, “Context-aware mobile health monitoring: Evaluation of different pat-tern recognition methods for classification of physical activity," in 30th Annual International Conference of the IEEE Engineering in Medicine and Biology Society, pp. 5250-5253, 2008. https://doi.org/10.1109/ IEMBS.2008.4650398

[15] C. Zhu and W. Sheng, "Human daily activity recognition in robot-assisted living using multi-sensor fusion," 2009 IEEE International Conference on Robotics and Automation, Kobe, 2009, pp. 2154-2159, doi: https://doi.org/10.1109/ROBOT.2009.5152756

[16] T. Mitchell, "Decision Tree Learning", in T. Mitchell, Machine Learning, the McGraw-Hill Companies, Inc., 1997, pp. 52-78.

[17] San-Segundo-Hernández, R., Blunck, H., Moreno-Pimentel, J., Stisen, A., \& Gil-Martín, M. (2018). Robust Human Activity Recognition using smartwatches and smartphones. Eng. Appl. Artif. Intell. 72, 190-202. https://doi.org/10.1016/j.engappai.2018.04.002

[18] E. Bulbul, A. Cetin and I. A. Dogru, "Human Activity Recognition Using Smartphones," 2018 2nd International Symposium on Multidisciplinary Studies and Innovative Technologies (ISMSIT), Ankara, 2018, pp. 1-6, doi: https://doi.org/10.1109/ISMSIT.2018.8567275.

[19] Z. He and L. Jin, "Activity recognition from acceleration data based on discrete cosine transform and svm," in IEEE International Conference on Systems, Man and Cybernetics, pp. 5041-5044, 2009. https://doi.org/10.1109/ICSMC.2009.5346042

[20] A. Khan, Y.-K. Lee, S. Lee, and T.-S. Kim, "A triaxial accelerometer-based physicalactivity recognition via augmented-signal features and a hierarchical recognizer," IEEE Trans. Inf. Technol. Biomed., vol. 14, no. 5, pp. 1166-1172, 2010. https://doi.org/10.1109/ TITB.2010.2051955

[21] Ong Vienna Lee, Ahmad Heryanto, Mohd Faizal Ab Razak, Anis Farihan Mat Raffei, Danakorn Nincarean Eh Phon, Shahreen Kasim, Tole Sutikno, “A malicious URLs detection system using optimization and machine learning classifiers", Indonesian Journal of Electrical Engineering and Computer Science, vol. 17, no. 3, pp. 1210-1214, March 2020. https://doi.org/10.11591/ijeecs.v17.i3.pp1210-1214

[22] Owais Mujtaba Khandy, Samad Dadvandipour, "Analysis of machine learning algorithms for character recognition: a case study on handwritten digit recognition", Indonesian Journal of Electrical Engineering and Computer Science, vol. 21, no. 1, pp. 574-581, January 2021. https://doi.org/10.11591/ijeecs.v21.i1.pp574-581 
[23] Arselan Ashraf, Teddy Surya Gunawan, Bob Subhan Riza, Edy Victor Haryanto, Zuriati Janin, "On the review of image and video-based depression detection using machine learning", Indonesian Journal of Electrical Engineering and Computer Science, vol. 19, no. 3, pp. 1677-1684, September 2020. https://doi.org/10.11591/ijeecs.v19.i3.pp1677-1684

[24] Rana Muhammad Adnan, Zhongmin Liang, Alban Kuriqi, Ozgur Kisi, Anurag Malik, Binquan Li, Fatemehsadat Mortazavizadeh, "Air temperature prediction using different machine learning models", Indonesian Journal of Electrical Engineering and Computer Science, vol. 22, no. 1, pp. 534-541, April 2021. https://doi.org/10.11591/ijeecs.v22. i1.pp534-541

[25] Monirul Islam, Mohammod Abul Kashem, Jia Uddin, "Fish survival prediction in an aquatic environment using random forest model", IAES International Journal of Artificial Intelligence (IJ-AI), vol. 10, no. 3, September 2021.

[26] Ong Wei Chuan, Nur Fadilah Ab Aziz, Zuhaila Mat Yasin, Nur Ashida Salim, Norfishah A. Wahab, "Fault classification in smart distribution network using support vector machine", Indonesian Journal of Electrical Engineering and Computer Science, vol. 18, no. 3, pp. 1148-1155, June 2020. https://doi.org/10.11591/ijeecs.v18.i3.pp1148-1155

[27] M. Tanaskuli, Ali N. Ahmed, Nuratiah Zaini, Samsuri Abdullah, Abdoulhdi A. Borhana, N. A. Mardhiah, Mathivanan Mathivanan, "Ozone prediction based on support vector machine", Indonesian Journal of Electrical Engineering and Computer Science, vol. 17, no. 3, pp. 1461-1466, March 2020. https://doi.org/10.11591/ijeecs.v17.i3.pp1461-1466

[28] Ni Made Gita Dwi Purnamasari, M. Ali Fauzi, Indriati Indriati, Liana Shinta Dewi, "Cyberbullying identification in twitter using support vector machine and information gain-based feature selection”, Indonesian Journal of Electrical Engineering and Computer Science, vol. 18, no. 3, pp. 1494-1500, June 2020. https://doi.org/10.11591/ijeecs.v18.i3.pp1494-1500

[29] Mohd Hatta Jopri, Abdul Rahim Abdullah, Jingwei Too, Tole Sutikno, Srete Nikolovski, Mustafa Manap, "Support-vector machine and naïve bayes based diagnostic analytic of harmonic source identification", Indonesian Journal of Electrical Engineering and Computer Science, vol. 20, no. 1, pp. 1-8, October 2020. https://doi.org/10.11591/ijeecs.v20.i1.pp1-8

[30] Naaman Omar, Adnan Mohsin Abdulazeez, Abdulkadir Sengur, Salim Ganim Saeed Al-Ali, "Fused faster RCNNs for efficient detection of the license plates", Indonesian Journal of Electrical Engineering and Computer Science, vol. 19, no. 2, pp. 874-982, August 2020. https://doi.org/10.11591/ijeecs.v19.i2.pp874-982

[31] Prabira Kumar Sethy, Nalini Kanta Barpanda, Amiya Kumar Rath, Santi Kumari Behera, "Rice false smut detection based on faster R-CNN", Indonesian Journal of Electrical Engineering and Computer Science, vol. 19, no. 3, pp. 1590-1595, September 2020. https://doi. org/10.11591/ijeecs.v19.i3.pp1590-1595

[32] Omar AlShorman, Buthaynah Alshorman, Fahed Alkahtani, "A review of wearable sensors-based monitoring with daily physical activity to manage type 2 diabetes", International Journal of Electrical and Computer Engineering (IJECE), vol. 11, no. 1, pp. 646-653, February 2021. https://doi.org/10.11591/ijece.v11i1.pp646-653

[33] Ding, G., Tian, J., Wu, J., Zhao, Q., \& Xie, L., "Energy efficient human activity recognition using wearable sensors", IEEE Wireless Communications and Networking Conference Workshops (WCNCW), pp. 379-383, 2018. https://doi.org/10.1109/WCNCW.2018.8368980

[34] J. Wu, S. Guo, H. Huang, W. Liu and Y. Xiang, "Information and Communications Technologies for Sustainable Development Goals: State-of-the-Art, Needs and Perspectives," in IEEE Communications Surveys \& Tutorials, vol. 20, no. 3, pp. 2389-2406, thirdquarter 2018, doi: https://doi.org/10.1109/COMST.2018.2812301.

[35] J. Wu, S. Guo, J. Li and D. Zeng, "Big Data Meet Green Challenges: Big Data Toward Green Applications," in IEEE Systems Journal, vol. 10, no. 3, pp. 888-900, Sept. 2016, doi: https://doi.org/10.1109/JSYST.2016.2550530. 
[36] J. Wu, S. Guo, J. Li and D. Zeng, "Big Data Meet Green Challenges: Greening Big Data," in IEEE Systems Journal, vol. 10, no. 3, pp. 873-887, Sept. 2016, doi: https://doi.org/10.1109/ JSYST.2016.2550538.

[37] R. Atat, L. Liu, J. Wu, G. Li, C. Ye and Y. Yang, "Big Data Meet Cyber-Physical Systems: A Panoramic Survey," in IEEE Access, vol. 6, pp. 73603-73636, 2018, doi: https://doi. org/10.1109/ACCESS.2018.2878681.

[38] Ahmed Al Marouf, Md. Ferdousur Rahman Sarker, Shah Md. Tanvir Siddiquee, "Recognizing Hand-based Actions based on Hip-Joint centered Features using KINECT", 2nd International Conference on Electrical \& Electronic Engineering (ICEEE), 27-29 December 2017, RUET, Rajshahi, Bangladesh. https://doi.org/10.1109/CEEE.2017.8412879

[39] I. Alghamdi, Mohammed. "Survey on Applications of Deep Learning and Machine Learning Techniques for Cyber Security.” International Journal of Interactive Mobile Technologies (iJIM) [Online], 14.16 (2020): pp. 210-224. Web. 1 Jul. 2021. https://doi.org/10.3991/ijim. v14i16.16953

[40] Alqudah, Yazan, Belal Sababha, Esam Qaralleh, \& Tarek Yousseff. "Machine Learning to Classify Driving Events Using Mobile Phone Sensors Data." International Journal of Interactive Mobile Technologies (iJIM) [Online], 15.02 (2021): pp. 124-136. Web. 1 Jul. 2021. https://doi.org/10.3991/ijim.v15i02.18303

[41] Samann, Fady, Adnan Mohsin Abdulazeez, \& Shavan Askar. "Fog Computing Based on Machine Learning: A Review." International Journal of Interactive Mobile Technologies (iJIM) [Online], 15.12 (2021): pp. 21-46. Web. 1 Jul. 2021. https://doi.org/10.3991/ijim. v15i12.21313

[42] Saraubon, Kobkiat, Nuttapong Wiriyanuruknakon, \& Natdanai Tangthirasunun. "Flashover Prevention System using IoT and Machine Learning for Transmission and Distribution Lines." International Journal of Interactive Mobile Technologies (iJIM) [Online], 15.11 (2021): pp. 34-48. Web. 1 Jul. 2021. https://doi.org/10.3991/ijim.v15i11.20753

\section{Authors}

Ashraful Alam is from the Department of Computer Science and Engineering of Daffodil International University (DIU). He is a potential researcher in the field of machine learning, deep learning, wearable sensors and artificial intelligence in healthcare.

Anik Das is from the Department of Computer Science and Engineering of Daffodil International University (DIU). He is a potential researcher in the field of machine learning, deep learning, data science and artificial intelligence in application areas.

Md. Shahriar Tasjid is from the Department of Computer Science and Engineering of Daffodil International University (DIU). He is a potential researcher in the field of machine learning, data science, wearable sensors and artificial intelligence. He possesses a great passion in cycling around the country.

Ahmed Al Marouf is currently working as a senior lecturer at the Department of Computer Science and Engineering of Daffodil International University (DIU). He is currently pursuing his Ph.D. in Computer Science at the Department of Computer Science of University of Calgary, Alberta, Canada. He is a potential researcher in the field of computational intelligence, mobile technologies and artificial intelligence in health science.

Article submitted 2021-07-01. Resubmitted 2021-08-06. Final acceptance 2021-08-06. Final version published as submitted by the authors. 